\title{
Pulpal and Periapical Response After Restoration of Deep Cavities in Dogs' Teeth With Filtek Silorane and Filtek Supreme XT Systems
}

\author{
E Ruiz-de-Castañeda • P Gatón-Hernández • EG Rodriguez \\ RAB Silva • P Nelson-Filho • LAB Silva
}

\begin{abstract}
Clinical Relevance
Both the silorane and methacrylate resin systems showed good tissue compatibility, suggesting that their placement in contact with deep dentin in clinical procedures may be appropriate.
\end{abstract}

Esther Ruiz-de-Castañeda, MS, Catalan Society of Dentistry and Stomatology, Academy of Medical Sciences and Health of Catalonia and Balears, Barcelona, Spain

Patricia Gatón-Hernández, PhD, School of Dentistry, University of Barcelona, Barcelona, Spain

Encarnación G. Rodriguez, PhD, School of Dentistry, University of Granada, Granada, Spain

Raquel A.B. Silva, PhD, School of Dentistry of Ribeirão Preto, University of São Paulo, Department of Pediatric Clinic, Preventive and Community Dentistry, Ribeirão Preto, SP, Brazil

*Paulo Nelson-Filho, PhD, School of Dentistry of Ribeirão Preto, University of São Paulo, Department of Pediatric Clinic, Preventive and Community Dentistry, Ribeirão Preto, SP, Brazil

Léa A.B. Silva, PhD, School of Dentistry of Ribeirão Preto, University of São Paulo, Department of Pediatric Clinic, Preventive and Community Dentistry, Ribeirão Preto, SP, Brazil

*Corresponding author: Avenida do Café, s/n Monte Alegre, Ribeirão Preto, SP 14040-904, Brazil; e-mail: nelson@forp. usp.br

DOI: $10.2341 / 11-341-\mathrm{L}$

\section{SUMMARY}

Objective: This study evaluated, histopathologically, the pulpal and periapical response to a silorane-based resin (Filtek Silorane) and a methacrylate-based nanoparticle resin (Filtek Supreme XT) in deep cavities in dogs, having zinc oxide and eugenol-based cement (ZOE) as a control.

Methods: The tooth/bone blocks were collected after 10 and 90 days and processed for microscopic analysis of the dentin, pulp, and periapical tissues using a score system. Data were analyzed statistically by Kruskal-Wallis and Dunn post-test $(\alpha=0.05)$.

Results: At 10 days, the pulp, connective tissue, and periodontal ligament showed normal characteristics. No resorption areas were observed. Both resins caused significantly less $(p<0.05)$ periapical and pulpal inflammatory 
response than ZOE. At 90 days, for all materials, the connective pulp tissue was healthy and dense, with a normal blood vessel system. The apical and periapical region had normal structure and thickness.

Conclusions: The use of the Filtek Silorane and the Filtek Supreme XT resins caused no adverse pulpal and periapical reactions after restoration of deep dentin cavities in vivo.

\section{INTRODUCTION}

Reactive components released from conventional composite resins, such as unreacted monomers, may induce toxicity or inflammatory tissue reactions depending on their aggressiveness ${ }^{1,2}$ and the amount of dentin remaining on the cavity floor. ${ }^{3}$ In contact with exposed pulp, adhesive systems may also unchain an unfavorable tissue reaction, causing pulp necrosis and apical periodontitis. ${ }^{4}$

The results of cell culture studies have shown that methacrylate and dimethacrylate monomers, commonly used in restorative polymeric technology, may affect the recruitment of leukocytes in inflammation sites by decreasing the expression of intercellular adhesion molecules ${ }^{5}$ and inducing enzymatic activity and expression of growth factors and cellular cytokines. ${ }^{6}$ In addition, resin monomers suppress the mitochondrial activity of macrophages and alter their inflammatory responses. ${ }^{7,8}$

In the last few years, methacrylate-based resins with modifications in their composition and structure have been developed, such as Filtek Supreme $\mathrm{XT}$. This resin is a nanocomposite recently launched by 3M ESPE (St Paul, MN, USA) that contains only nanoparticles and nanoclusters as inorganic fillers. The nanoparticles are monodispersed, nonagglomerated silica particles, while the nanoclusters are spheroidal agglomerates consisting of nano-sized silica and zirconia particles. The micron-sized porous cluster is infiltrated with silane coupling agents to allow chemical bonding with the organic matrix. Although adequate physical properties have been attributed to these resins (eg, improved surface polishing), ${ }^{9}$ toxic effects have been observed in cell cultures. $^{10}$

On the other hand, the occurrence of polymerization shrinkage and the release of chemical products from methacrylate-based systems have led to the development of resinous materials with improvement of polymerization technology, wear resistance, esthetics, and adhesive properties. ${ }^{11}$ Recently, a silorane-based composite resin with a distinctive polymerization characteristic to reduce polymerization shrinkage has been introduced to the market (Filtek Silorane, 3M ESPE). Silorane matrix is formed by the cationic ring-opening polymerization of the silorane monomers. A silorane molecule represents a hybrid that is made of both siloxane and oxirane structural moieties. Silorane technology has afforded a highly hydrophobic restorative material with reduced polymerization shrinkage, ${ }^{12,13}$ more balanced volumetric stress, higher ambient light stability, ${ }^{12}$ and insolubility in biologic fluids. ${ }^{14}$

Although several recent studies have evaluated physicochemical properties of silorane-based resins, ${ }^{13-17}$ research on their biologic effects is quite limited, being based mostly on in vitro and cell culture studies. $^{5,10,18}$ To the best of our knowledge, the in vivo pulpal and periapical response to these new restorative systems has not yet been investigated.

The present study evaluated the in vivo pulpal and periapical response to a silorane-based resin system (Filtek Silorane) and a methacrylate-based nanoparticle resin (Filtek Supreme XT) after restoration of deep cavities prepared in dogs' teeth.

\section{MATERIALS AND METHODS}

All experiments were conducted strictly in accordance with the guidelines of the university's Ethics Committee for the Care and Use of Laboratory Animals. The study design and histologic parameters for pulpal and periapical tissue reaction evaluation were in accordance with the protocol recommended by the International Organization for Standardization for biologic evaluation of dental materials (ISO standard 7405:1997). ${ }^{19}$

The second and third maxillary premolars and the second, third, and fourth mandibular premolars of five mongrel dogs aged 12-18 months and weighing $15 \mathrm{~kg}$ on average were selected for the study, providing 50 teeth. The dogs were maintained in quarantine and received vermifuges, vitamin supplements, and antirabic and triple vaccines to put them in adequate health conditions for the experiment. The dogs were housed with free access to water and standard lab chow during the whole course of the study.

The 50 teeth were randomly distributed into six groups: groups I and IV (experimental) ( $\mathrm{n}=10$ teeth/ group)-restoration with Filtek Silorane system (3M ESPE) for 10 and 90 days, respectively; groups II and V (experimental) ( $\mathrm{n}=10$ teeth/group)—restoration with Filtek Supreme XT system (3M ESPE) for 
10 and 90 days, respectively; groups III and VI (control) ( $\mathrm{n}=5$ teeth/group)-restoration with zinc oxide and eugenol-based cement (ZOE) (Caulk IRM Intermediate Restorative Material, Dentsply, Milford, DE, USA) for 10 and 90 days, respectively.

The animals were preanesthetized with an endovenous injection of levomepromazine (Neozine; $1 \mathrm{mg} /$ kg body weight; Aventis Pharma, São Paulo, SP, Brazil) 15 minutes before the operative procedures and then anesthetized with an endovenous injection of tiletamine hydrochloride and zolazepam hydrochloride $(0.1 \mathrm{~mL} / \mathrm{kg}$ body weight; Zoletil 50 , Virbac do Brazil Ind e Com, São Paulo, Brazil) to facilitate the passage of an endotracheal tube. Inhalation anesthesia with Isoflurane (Abbott Laboratories, SaintLaurent, Quebec, Canada) was delivered using an inhalation anesthesia apparatus (Takaoka KT-20, Takaoka Ind e Com, São Paulo, Brazil). Throughout the duration of the operative procedures, the animals were maintained on isotonic saline solution $(0.9 \% \mathrm{NaCl}$; Glicolabor Indústria Farmacêutica, Ribeirão Preto, SP, Brazil).

Standardized radiographs of the teeth to be treated were taken using custom-made film-holding devices and size 2 periapical films (Ultraspeed, Eastman Kodak Co, Rochester, NY, USA). An exposure time of 1 second was used with the x-ray equipment (Heliodent, Siemens, Siemens Medical Systems, Iselin, NJ, USA) operating at $60 \mathrm{kVp}$ and $10 \mathrm{~mA}$. The exposed films were processed in an automated processing machine.

All teeth were isolated with a rubber dam, and the operative field was disinfected with $3 \%$ hydrogen peroxide followed by the application of $1 \%$ chlorhexidine gluconate. After pumice/rubber cup prophylaxis, Class V cavities were prepared on the buccal surface of each tooth with a sterile \#1015 bur (KG Sorensen, São Paulo, SP, Brazil) in a high-speed handpiece under abundant air/water spray cooling. Cavity depth was standardized based on the size of the active part of the \#1015 bur and the mean thickness of the enamel and dentin of canine teeth, leaving a remaining dentin thickness in the cavity floor between 0.5 and $1.0 \mathrm{~mm}$. The burs were used with intermittent movements and without pressure to avoid overheating. Burs were replaced every four preparations to maintain a good cutting efficiency. The cavities were abundantly irrigated with sterile saline (Glicolabor Indústria Farmacêutica Ltda, Ribeirão Preto, SP, Brazil) to remove debris and enamel and dentin chips, and were dried with cotton pellets.
The cavities were restored with the materials corresponding to each group (I to VI), according to the manufacturers' instructions. In groups I and IV, Filtek Silorane self-etch primer was applied actively for 15 seconds with a disposable microbrush tip, followed by gentle air dispersion and light curing for 10 seconds with a halogen source (Ultralux Electronic, Dabi Atlante, Ribeirão Preto, SP, Brazil) with light intensity of $450 \mathrm{~mW} / \mathrm{cm}^{2}$ as measured with a curing radiometer (Demetron, Kerr Corp, Danbury, CT, USA). A layer of Filtek Silorane adhesive bond was applied, followed by gentle air thinning and 10 seconds of light curing. The cavities were restored with the composite resin using an incremental technique. In groups II and V, Scotchbond Etchant was applied for 15 seconds, rinsed for 10 seconds, and excess water was blotted using a cotton pellet. Two to three consecutive coats of Adper Single Bond 2 Plus Adhesive were applied for 15 seconds with gentle agitation using a fully saturated applicator, gently air thinned for 5 seconds to evaporate solvent, and light-cured for 10 seconds. In groups III and VI, $\mathrm{ZOE}$ was prepared and taken to the cavities following the conventional technique.

As all variables should be evaluated in the same animal and in the different dental quadrants, each hemiarch was alternately subjected to the different experimental protocols. Each hemiarch was radiographed, and the animals were maintained under observation during the whole experiment.

At the predetermined experimental periods (10 and 90 days postoperatively), the teeth of each group were radiographed, and the animals were euthanized with a lethal intravenous overdose of sodium pentobarbital. The maxilla and mandible were dissected, reduced in volume, and the restored teeth were removed in blocks (tooth/bone) using a water-cooled diamond saw. The specimens were fixed in a $10 \%$ buffered formalin solution for 48 hours at room temperature, demineralized in $10 \%$ EDTA, $\mathrm{pH}$ 7.4, during approximately 30 days, subjected to routine histologic processing, and embedded in paraffin.

Five-micrometer-thick slides were serially cut longitudinally in a buccolingual direction and were stained with hematoxylin and eosin, Mallory's Trichrome, and Brown and Brenn staining techniques. A single calibrated observer blinded to the treatment groups performed all examinations in a subjective manner using an optical microscope (Axio Imager.M1, Carl Zeiss, Gottingen, Germany) coupled to a digital camera (AxioCam MRc5, Carl Zeiss). The pulpal and periapical response to the tested 
materials was evaluated in front of the whole extension of the pulpal wall of the cavity based on the histopathologic parameters described by Silva and others. ${ }^{4,20}$ The characteristics of dentin, pulp, and periapical tissues were evaluated according to the following scores: Odontoblast layer: 1 - present, 2 - absent; Inflammatory pulp response: 1 - absent, 2 mild, 3 - moderate, 4 - severe; Mineralized tissue resorption: 1 - absent, 2 - mild, 3 - moderate, 4 severe; and Bacteria: 1 - present, 2 - absent. The data were analyzed statistically by Kruskal-Wallis and Dunn post-test using Graph Pad Prism 5.0 (Graph Software Inc, San Diego, CA, USA). A significance level of $5 \%$ was set for all analyses.

The thickness of remaining dentin between the pulpal wall of the cavity and the pulp chamber roof was measured in micrometers in images obtained from three slides per specimen at $5 \times$ magnification. In all teeth, the remaining dentin thickness was standardized in three regions: at half of the length of the pulpal wall of the cavity and at two regions equidistant between this point and the lateral wall in the right and in the left.

For the radiographic analysis, three calibrated observers (kappa $=0.9$ ) examined the periapical radiographs taken before, 10 days and 90 days after the operative procedures as to the presence or absence of lamina dura, areas of periapical bone rarefaction, and internal and external root resorption.

\section{RESULTS}

After the 10- and 90-day experimental periods, all fillings were still present in all teeth of the six groups. The mean thickness of remaining dentin between the pulpal wall of the cavity and the pulp chamber roof was $530 \mu \mathrm{m}$ (standard deviation $=225$ $\mu \mathrm{m})$.

\section{Groups I and II: Filtek Silorane and Filtek Supreme XT (10 Days)}

As these groups presented similar histopathologic features, the results are presented together as follows. In all 10 teeth, the pulp tissue was normal, exhibiting an odontoblast layer with vacuolization areas under the remaining dentin layer. In the superficial portion of the pulp, the connective tissue was normal with mild vascular congestion and spindle-shaped fibroblasts. The apical region was normal, with a uniform cementum layer and cementoblasts on the entire surface. The periodontal ligament was normal with intense presence of fibers and absence of inflammatory cells. There were no areas of mineralized tissue resorption. Bacteria were not detected.

\section{Group III: ZOE (10 Days)}

In the five teeth of this group the histopathologic features were similar to those described in groups I and II, except for discrete presence of mononuclear inflammatory cell in the pulp core, close to the remaining dentin layer. Bacteria were not detected.

\section{Groups IV and V: Filtek Silorane and Filtek Supreme XT (90 Days)}

As these groups presented similar histopathologic features, the results are presented together as follows. One tooth of group IV and two teeth of group V had accidental pulp exposure during cavity preparation, reducing the number of teeth to nine and eight, respectively. In all teeth of both groups, the pulp tissue was healthy, exhibiting a normal odontoblast layer underneath the remaining dentin layer and along the root canal walls. The connective tissue was dense, with a normal vascular system. The apical and periapical region had normal structure and thickness. There were no areas of mineralized tissue resorption or inflammatory cells. Figures 1 and 2 present sets of panels of microscopic images of these groups. The histopathologic features observed at 90 days are also representative of those observed at 10 days. Bacteria were not detected, as illustrated in Figures $1 \mathrm{D}$ and 2C.

\section{Group VI: ZOE (90 Days)}

At 90 days, the five teeth restored with $\mathrm{ZOE}$ presented similar histopathologic features to those observed in the 10-day period, except for the absence of inflammatory cells in the pulp tissue adjacent to the remaining dentin.

\section{Statistical Analysis}

Statistically significant difference was found only for periapical and pulpal inflammatory response at 10 days: Filtek Silorane and Filtek Supreme XT had a similar behavior and both materials differed significantly from ZOE $(p<0.05)$. The distribution of teeth according to the scores attributed to the histopathologic parameters in the four groups and the statistical analysis are presented in Table 1.

\section{Radiographic Analysis}

There were no alterations regarding the integrity of the lamina dura, presence of areas of periapical bone rarefaction, or presence of internal or external root 


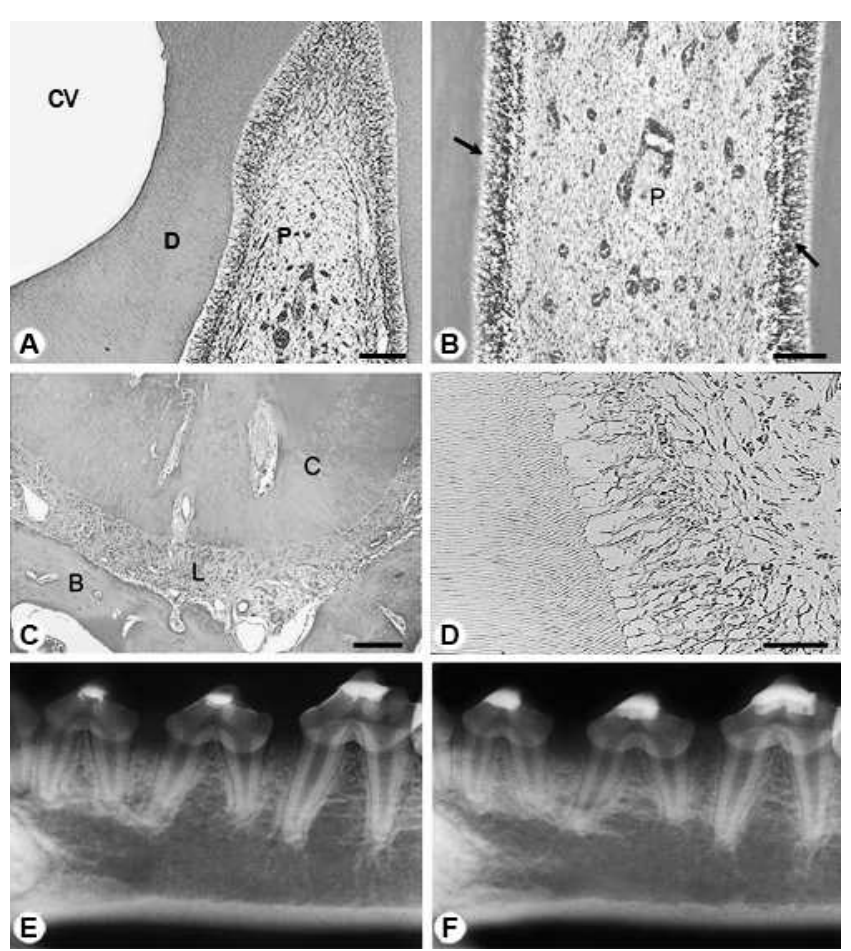

Figure 1. Filtek Silorane system (90 days). (A): Cavity (CV), normal dentin (D), and pulp tissue (P). Hematoxylin Eosin (H.E.), 100x magnification. Scale bar: $50 \mu \mathrm{m}$. $(B)$ : Pulp tissue $(P)$ with accentuate fibroblast population, vessels, and odontoblast layer (arrows). H.E., 200× magnification. Scale bar: $20 \mu \mathrm{m}$. (C): Apical and periapical region. Normal periodontal ligament (L), cementum (C), and alveolar bone (B). H.E., 100× magnification. Scale bar: $20 \mu \mathrm{m}$. (D): Representative Brown- and Brenn-stained images illustrating the absence of bacteria. 400× magnification. Scale bar: $50 \mu \mathrm{m}$. (E): Radiographic image immediately after the operative procedures. (F): Radiographic image 90 days after the operative procedures, showing no alterations.

resorption in any specimen at either of the two experimental periods.

\section{Pulp Exposure (Groups IV and V)}

In the teeth restored with Filtek Silorane and Filtek Supreme XT in which the pulp was accidentally exposed during cavity preparation, the odontoblast layer was absent and the pulp tissue, if present, was necrotic along the canal extension. In the root apex, cementum surface was free of cementoblasts and had a markedly irregular appearance due to resorption. The periodontal ligament was widened, exhibiting intense fiber dissociation, edema, and mononuclear and polymorphonuclear inflammatory cell infiltrate. Microorganisms were not observed.

\section{DISCUSSION}

As restorations are frequently placed in deep cavities, the transdentinal diffusion of products
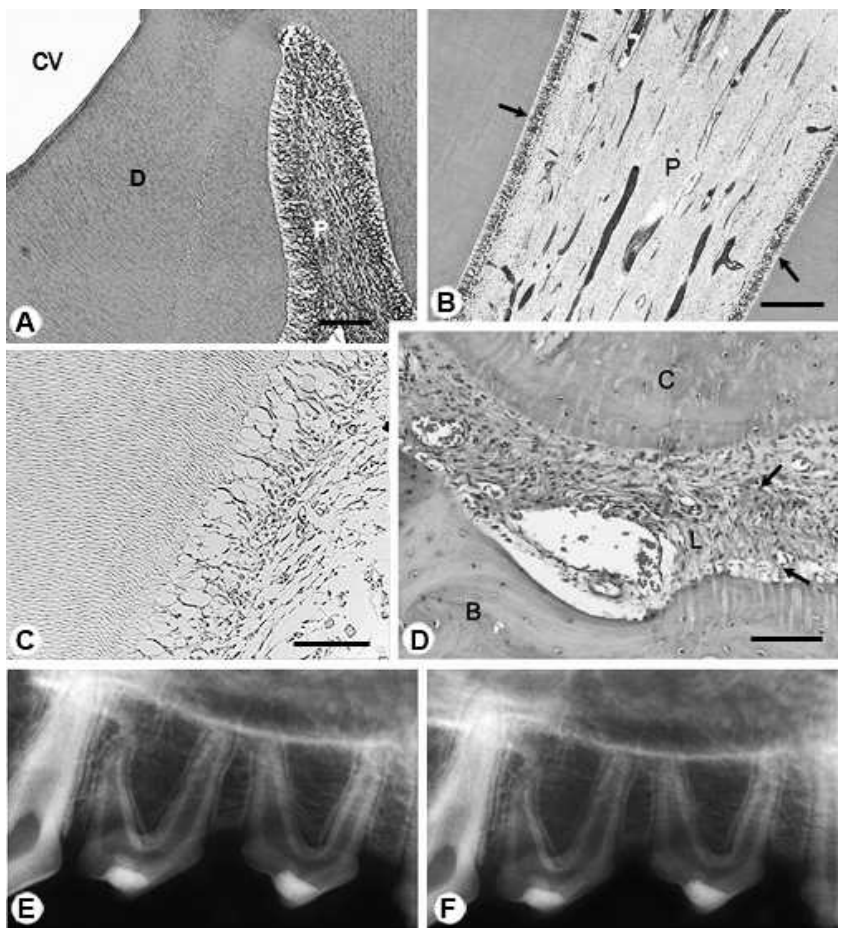

Figure 2. Filtek Supreme XT (90 days). (A): Cavity (CV), normal dentin (D), and pulp tissue (P). H.E., $100 \times$ magnification. Scale bar: $50 \mu \mathrm{m}$. (B): Normal pulp (P) and odontoblast layer (arrows). H.E., $100 \times$ magnification. Scale bar: $20 \mu \mathrm{m}$. (C): Representative Brownand Brenn-stained images illustrating the absence of bacteria. 400X magnification. Scale bar: $50 \mu \mathrm{m}$. (D): Interwoven collagen fibers (arrows) in the periodontal ligament (L), normal cementum (C), and alveolar bone (B). H.E., 200× magnification. Scale bar: $20 \mu \mathrm{m}$. (E): Radiographic image immediately after the operative procedures. (F): Radiographic image 90 days after the operative procedures, showing no alterations.

released from restorative materials towards the dental pulp may cause pathologic alterations, depending on the size of the molecule, material composition, the surface available for diffusion, dentin tubule permeability, and remaining dentin thickness. For these reasons, deep cavities were prepared to evaluate the pulpal and periapical tissue compatibility of Filtek Supreme and Filtek Silorane resin systems.

Pulp reaction to cavity preparation may vary from a mild inflammatory response associated with slight tissue disorganization to partial pulp necrosis or complete pulp breakdown. Several factors such as air drying of exposed dentin, heat generation by continuous cutting during cavity preparation, or inadequate water cooling, among others, have been considered as responsible for eliciting pulp damage. $^{21,22}$ In the present study, the cavities were prepared under copious air/water spray cooling and with intermittent movements using high-speed burs 


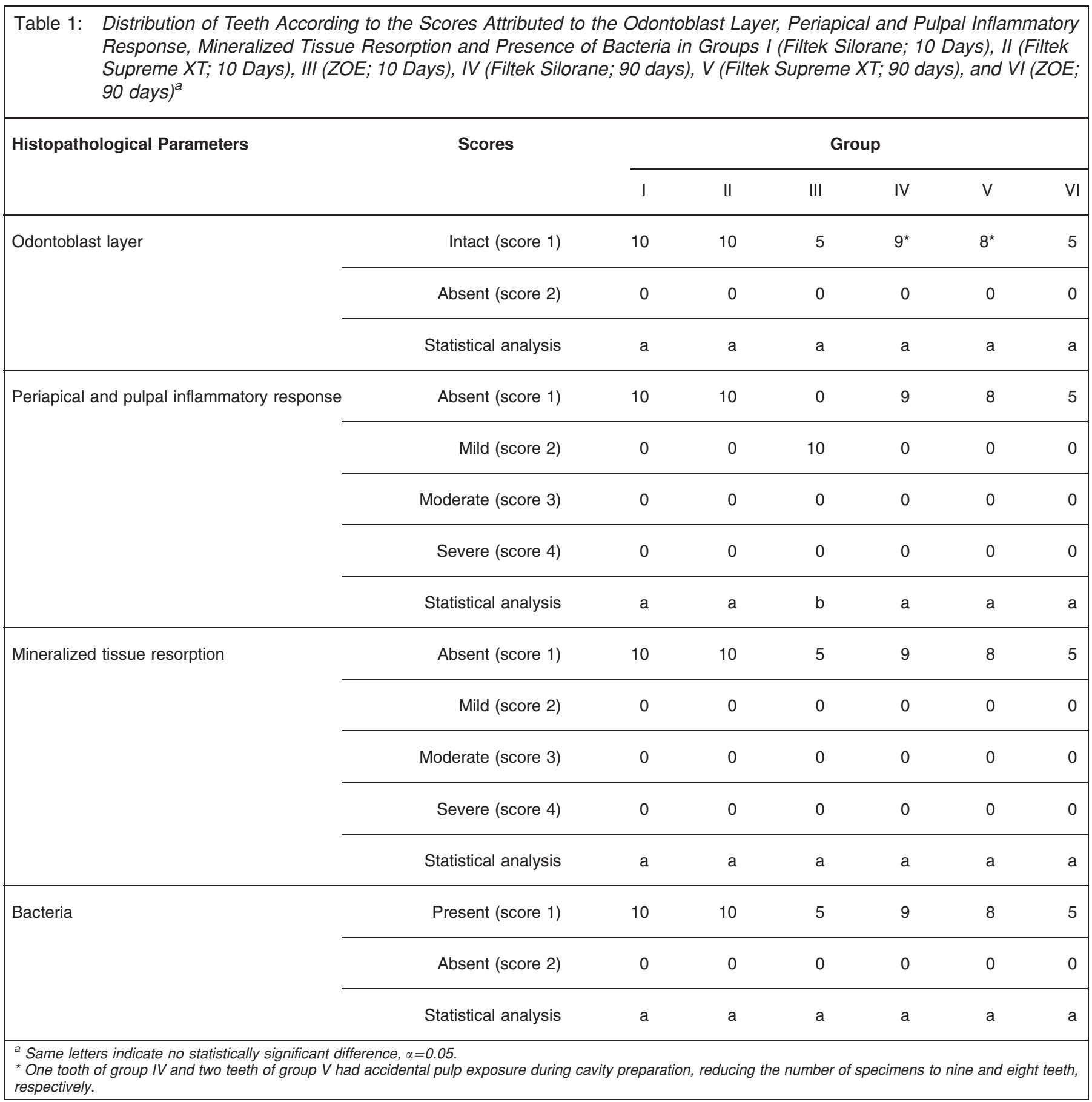

with good cutting efficiency. These cautions probably minimized the thermal aggressions, since no significant pathologic alterations were found in the 10-day period in either of the groups.

Another parameter that may have an impact on the results is the evaluation period. In the present study, the restorations were made at two different time points of an 80-day interval (10 and 90 days) to allow the collection of the teeth at the same time. The rationale for this sampling schedule design was primordially to have restorations of both experimental periods placed in the same animal. Another methodological design would be to perform the restorative work for all animals at baseline and then conduct the sacrifice/collection of teeth according to 
the preset schedule at 10 days and 90 days. However, the use of this sampling schedule (ie, place the restorations in different animals allocated to each experimental period) would have some important implications that were taken into consideration during preparation of the study. Firstly, the fact that dogs are not isogenic animals would bring more interferences and biases to the results. Having a material placed in one animal sacrificed at 10 days and having the same material placed in a different animal sacrificed at 90 days would disregard the individual factors inherent to each animal, as every animal responds differently to the experimental conditions and evaluation times. Secondly, there would be ethical implications because the number of animals to be sacrificed for collection of teeth at 10 days and then at 90 days should be considerably increased. Finally, the overall costs of the study would be significantly increased.

\section{Filtek Supreme XT}

In the last few years, methacrylate-based resins with modifications in their composition and structure have been developed, such as the nanocomposite Filtek Supreme XT. Although these resins have better physical properties, their cytotoxicity in cell cultures has been demonstrated. ${ }^{10}$ Filtek Supreme $\mathrm{XT}$ has also been shown to have a high monomer release rate $^{23}$ and cytotoxicity to L929 fibroblasts. ${ }^{24}$ Although the characterization of tissue response is a key factor to substantiate the clinical use of new dental materials, no histopathologic study has yet evaluated pulp response in vivo after restoration of deep cavities with Filtek Supreme XT resin. In the present study, there was good pulpal and periapical response to the placement of this material in deep cavities. This possibly occurred because Filtek Supreme XT is composed of nanoparticles and nanoclusters, ${ }^{9}$ which may reduce the interstitial space and, consequently, the amount of organic matrix capable of releasing methacrylates, thus causing less tissue irritation than conventional methacrylate-based resins.

\section{System Filtek Silorane}

According to Ilie and Hickel, ${ }^{16}$ the macromechanical, micromechanical, and nanomechanical properties of silorane-based resins are comparable to those of methacrylate-based resins. Cell culture studies investigating the biologic properties of siloranebased resins have shown no cytotoxic effects ${ }^{5}$ and low mutagenic potential. ${ }^{18}$ Another study ${ }^{10}$ evaluating the in vitro cytotoxicity of different resins in
Balb/c 3T3 mouse fibroblast cell cultures during eight weeks found that the methacrylate-free resin Hermes, precursor of Filtek Silorane, presented lower cytotoxicity than methacrylate-based resins and was similar to that of Teflon (control). However, to date, there is no published study evaluating the in vivo pulpal and periapical response to the use of Filtek Silorane as a restorative material in deep cavities. In the present study, the pulp tissue had an adequate response to the placement of this material in deep dentin at both evaluation periods. Materials with new polymerization modes are promising for reducing the release of uncured components and, consequently, their cytotoxicity. ${ }^{10}$ The results of the present study may have great clinical significance because, in addition to its claimed low polymerization shrinkage, which improves its clinical performance, Filtek Silorane showed tissue compatibility when placed in contact with deep dentin in vivo.

\section{Pulp Exposure (Groups IV and V)}

It has been demonstrated that, even in the absence of bacterial contamination, direct pulp capping with adhesive systems causes an inflammatory reaction, ${ }^{4,25}$ disruption, or disappearance of the odontoblast layer, pulp necrosis, ${ }^{26}$ and absence of dentin barrier formation. ${ }^{27}$ Gerzina and Hume ${ }^{28}$ observed that the hybrid layer produced with methacrylatebased adhesive systems cannot be considered an impermeable barrier because it does not prevent the diffusion of monomers to the pulp. In our study, the teeth in which cavities with accidental pulp exposure were restored with Filtek Supreme XT and Filtek Silorane presented pulp necrosis, periodontal ligament widening, edema, intense mononuclear and polymorphonuclear inflammatory cell infiltrate, bone resorption, and cemental resorption, even in the absence of microorganisms. These results indicate that these materials should not be placed in direct contact with exposed pulp.

\section{ZOE}

The teeth restored with $\mathrm{ZOE}$ did not present significant pulpal alterations, except for the presence of inflammatory cell infiltrate in the 10-day period, which was more intense that that observed in the teeth restored with the resins. These results are in agreement with those of Murray and others $^{29}$ who reported that the use of $\mathrm{ZOE}$ for indirect pulp capping did not cause intense pulp reactions. 


\section{CONCLUSION}

Based on the findings of this in vivo study, it may be concluded that the use the silorane-based (Filtek Silorane) and the methacrylate-based nanoparticle (Filtek Supreme XT) resins caused no adverse pulpal and periapical reactions when placed in deep dentin cavities. Further research at other research levels and with longer evaluation periods, including clinical trials, are needed to support the clinical use of these materials.

\section{Conflict of Interest}

The authors of this manuscript certify that they have no proprietary, financial, or other personal interest of any nature or kind in any product, service, and/or company that is presented in this article.

(Accepted 19 March 2012)

\section{REFERENCES}

1. Braga RR, \& Ferracane JL (2004) Alternatives in polymerization contraction stress management Critical Reviews in Oral Biology and Medicine 15(3) 176-184.

2. Pfeifer CS, Ferracane JL, Sakaguchi RL, \& Braga RR (2008) Factors affecting photopolymerization stress in dental composites Journal of Dental Research 87(11) 1043-1047.

3. Pameijer CH, Stanley HR, \& Ecker G (1991) Biocompatibility of a glass ionomer luting agent. 2. Crown cementation American Journal of Dentistry 4(3) 134-141.

4. Silva LAB, Freitas AC, Carvalho FK, Queiroz AM, Nelson-Filho P, \& Porto-Neto ST (2009) Direct pulp capping with a self-etching adhesive system: Histopathologic evaluation in dogs' teeth Oral Surgery, Oral Medicine, Oral Pathology, Oral Radiology, and Endodontics 108(1) e34-e40.

5. Kostoryz EL, Tong PY, Strautman AF, Glaros AG, Eick JD, \& Yourtee DM (2001) Effects of dental resins on TNFalpha-induced ICAM-1 expression in endothelial cells Journal of Dental Research 80(9) 1789-1792.

6. Gregson KS, Terrence O’Neill J, Platt JA, \& Jack Windsor $\mathrm{L}$ (2008) In vitro induction of hydrolytic activity in human gingival and pulp fibroblasts by triethylene glycol dimethacrylate and monocyte chemotatic protein-1 Dental Materials 24(11) 1461-1467.

7. Rakich DR, Wataha JC, Lefebvre CA, \& Weller RN (1999) Effect of dentin bonding agents on the secretion of inflammatory mediators from macrophages Journal of Endodontics 25(2) 114-117.

8. Rakich DR, Wataha JC, Lefebvre CA, \& Weller RN (1998) Effects of dentin bonding agents on macrophage mitochondrial activity Journal of Endodontics 24(8) 528-533.

9. Mitra SB, Wu D, \& Holmes BN (2003) An application of nanotechnology in advanced dental materials Journal of the American Dental Association 134(10) 1382-1390.

10. Brackett MG, Bouillaguet S, Lockwood PE, Rotenberg S, Lewis JB, Messer RL, \& Wataha JC (2007) In vitro cytotoxicity of dental composites based on new and traditional polymerization chemistries Journal of Biomedical Materials Research. Part B, Applied Biomaterials 81(2) 397-402.

11. Bouillaguet S (2004) Biological risks of resin-based materials to the dentin-pulp complex Critical Reviews in Oral Biology and Medicine 15(1) 47-60.

12. Weinmann W, Thalacker C, \& Guggenberger R (2005) Siloranes in dental composites. Dental Materials 21(1) 68-74.

13. Lien W, \& Vandewalle KS (2010) Physical properties of a new silorane-based restorative system Dental Materials 26(4) $337-344$.

14. Eick JD, Kotha SP, Chappelow CC, Kilway KV, Giese GJ, Glaros AG, \& Pinzino CS (2007) Properties of siloranebased dental resins and composites containing a stressreducing monomer Dental Materials 23(8) 1011-1017.

15. Bagis YH, Baltacioglu IH, \& Kahyaogullari S (2009) Comparing microleakage and the layering methods of silorane-based resin composite in wide Class II MOD cavities Operative Dentistry 34(5) 578-585.

16. Ilie N, \& Hickel R (2009) Macro-, micro- and nanomechanical investigations on silorane and methacrylatebased composites. Dental Materials 25(6) 810-819.

17. Kopperud HM, Schmidt M, \& Kmildn IS (2010) Elution of substances from a silorane-based dental composite European Journal of Oral Sciences 118(1) 100-102.

18. Schweikl H, Schmalz G, \& Weinmann W (2004) The induction of gene mutations and micronuclei by oxiranes and siloranes in mammalian cells in vitro Journal of Dental Research 83(1) 17-21.

19. ISO Standards (1997) ISO 7405: Preclinical evaluation of biocompatibility of medical devices used in dentistry Tests methods for dental materials. International Organization for Standardization Geneva. Switzerland.

20. Silva LAB, Leonardo MR, Oliveira DSB, Silva RAB, Queiroz AM, Gatón-Hernández P, \& Nelson-Filho P (2010) Histopathological evaluation of root canal filling materials for primary teeth Brazilian Dental Journal 21(1) 38-45.

21. Goodis HE, Schein B, \& Stauffer P (1988) Temperature changes measured in vivo at the dentinoenamel junction and pulpodentin junction during cavity preparation in the Macaca fascicularis monkey Journal of Endodontics 14(7) 336-339.

22. Wisithphrom K, Murray PE, About I, \& Windsor LJ (2006) Interactions between cavity preparation and restoration events and their effects on pulp vitality International Journal of Periodontics \& Restorative Dentistry 26(6) 596-605.

23. Polydorou O, König A, Hellwig E, \& Kümmerer K (2009) Long-term release of monomers from modern dentalcomposite materials European Journal of Oral Sciences 117(1) 68-75.

24. Franz A, Konradsson K, König F, Van Dijken JW, \& Schedle A (2006) Cytotoxicity of a calcium aluminate cement in comparison with other dental cements and resin-based materials Acta Odontologica Scandinavica 64(1) $1-8$. 
25. Koliniotou-Koumpia E, \& Tziafas D (2005) Pulpal responses following direct pulp capping of healthy dog teeth with dentine adhesive systems Journal of Dentistry 33(8) 639-647.

26. Pameijer CH, \& Stanley HR (1998) The disastrous effects of the "total etch" technique in vital pulp capping in primates American Journal of Dentistry 11(Special No) S45-S54. Erratum in: American Journal of Dentistry 11(3) 148.

27. Elias RV, Demarco FF, Tarquinio SB, \& Piva E (2007) Pulp responses to the application of a self-etching adhesive in human pulps after controlling bleeding with sodium hypochlorite Quintessence International $\mathbf{3 8 ( 2 )}$ e67-e77.

28. Gerzina TM, \& Hume WR (1996) Diffusion of monomers from bonding resin-resin composite combinations through dentine in vitro Journal of Dentistry 24(1-2) 125-128.

29. Murray PE, About I, Franquin JC, Remusat M, \& Smith AJ (2001) Restorative pulpal and repair responses Journal of the American Dental Association 132(4) 482491. Erratum in: Journal of the American Dental Association 132(8) 1095. 